Access to this work was provided by the University of Maryland, Baltimore County (UMBC) ScholarWorks@UMBC digital repository on the Maryland Shared Open Access (MD-SOAR) platform.

Please provide feedback

Please support the ScholarWorks@UMBC repository by emailing scholarworks-group@umbc.edu and telling us what having access to this work means to you and why it's important to you. Thank you. 


\title{
Linkages of Calcium Induced Calcium Release in a Cardiomyocyte Simulated by a System of Seven Coupled Partial Differential Equations
}

\author{
Gerson C. Kroiz, Carlos Barajas, Matthias K. Gobbert and Bradford E. Peercy \\ (\{gkroiz1,barajasc,gobbert,bpeercy\}@umbc.edu) \\ Department of Mathematics and Statistics, \\ University of Maryland, Baltimore County, \\ 1000 Hilltop Circle, Baltimore, MD 21250 \\ Cardiac arrhythmias affect millions of adults in the U.S. each year. This \\ irregularity in the beating of the heart is often caused by dysregulation \\ of calcium in cardiomyocytes, the cardiac muscle cell. Cardiomyocytes \\ function through the interplay between electrical excitation, calcium sig- \\ naling, and mechanical contraction, an overall process known as calcium \\ induced calcium release (CICR). A system of seven coupled non-linear \\ time-dependent partial differential equations (PDEs), which model phys- \\ iological variables in a cardiac cell, link the processes of cardiomyocytes. \\ Through parameter studies for each component system at a time, we create \\ a set of values for critical parameters that connect the calcium store in the \\ sarcoplasmic reticulum, the effect of electrical excitation, and mechanical \\ contraction in a physiologically reasonable manner. This paper shows the \\ design process of this set of parameters and then shows the possibility to \\ study the influence of a particular problem parameter using the overall \\ model.
}

Keywords: Cardiac Arrhythmia, Calcium Induced Calcium Release, Reaction Diffusion Equations, Finite Volume Method, Parallel Computing

\section{INTRODUCTION}

The leading cause of death in the United States is currently heart disease [ $\left.\mathrm{M}^{+} 14\right]$. In order to continue searching for methods to combat heart disease, it is vital that the heart and its underlying processes are understood with greater depth. The importance of having a greater understanding of the heart provides the motivation for this research.

The line of work of this project focuses on a single cardiac cell and uses a mathematical model in order to represent the electrical excitation, calcium signaling, and mechanical contraction components of a cardiomyocyte. The original model for calcium induced calcium release (CICR) was introduced by Izu and co-workers [IMBW01, IWB01] with a three variable model and included only calcium signaling. This original model comprises the heart of the Calcium Signaling component of the system indicated in Figure 1 (a). Figure 1 (b) sketches the domain of the simulation. We use an hexahedron elongated in the $z$-direction for the shape of a cell, since the focus of CICR research is on estimating reasonable physiological parameter values. A key of the model[IMBW01, IWB01] is to place the calcium release units (CRUs) on a regular lattice of size, for instance, $15 \times 15 \times 31=6,975$ throughout the cell; Figure 1 (b) shows three $z$-planes of $3 \times 3$ CRUs as example. At these many locations, calcium ions are released from the calcium store in the sarcoplasmic reticulum (SR) into the cytosol of the cell, and CRUs can open ('fire') and close repeatedly over time[IMBW01, IWB01]. 


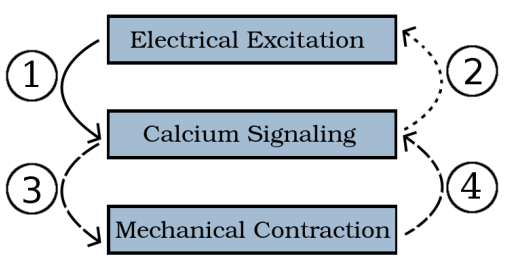

(a)

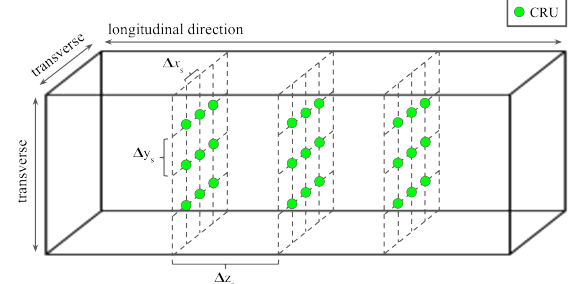

(b)

Figure 1. (a) The three components of the model and their links labeled (1) to (4). (b) The CRU lattice with spacings $\Delta x_{s}, \Delta y_{s}$, $\Delta z_{s}$ throughout the three-dimensional cell.

The model was extended for the first time to include the Electrical Excitation component in Figure 1 (a) $\left[\mathrm{ADFI}^{+} 15\right]$, which implemented a one-way interaction from electrical excitation to calcium signaling indicated by link (1) in Figure 1 (a). Studies with six variables in $\left[\mathrm{ABM}^{+} 16\right]$ extended the coupling to include a two-way cycle between electrical excitation and calcium signaling by incorporating both links (1) and (2) in Figure 1 (a). The 2016 paper $\left[\mathrm{ABM}^{+} 16\right]$ also introduced the formulation of the complete eight variable model for all components in Figure 1 (a), but not all model variables were used in the simulations, and the studies did not incorporate the mechanical system.

The most recent work in $\left[\mathrm{DFL}^{+} 17 \mathrm{a}, \mathrm{DFL}^{+} 17 \mathrm{~b}\right]$ studies the introduction of the Mechanical Contraction component in Figure 1 (a) by activating the links (3) and (4) in Figure 1 (a). This is facilitated by adding a buffer species whose concentration can be related to the contraction of the cell. Thus, this work uses a model with seven variables to represent the excitation-contraction coupling (ECC) occurring in the cardiomyocyte, in which CICR is the mechanism through which electrical excitation is coupled with mechanical contraction through calcium signaling. The initial results for the newly extended model when studying model in its entirety make simulations promising in their predictive capability.

From the previous work, the linkage of the mechanical contraction was existent, but not physiologically correct with the previous set of parameters. The resulting simulations may not have provided realistic behaviors of a cardiac muscle cell. As such, it is important to create a set of parameters with physioligcally reasonable simulations. To do this, we reduce the seven variable model to the three variable model from [IMBW01, IWB01] by setting key parameters to zero. This removes several CICR components: the calcium store in the SR, electrical excitation, and mechanical contraction. By this process, the additional CICR components are negated as they all reduce to zero. From this base case, we reintroduce physiologically reasonable values for these parameters in successive order. First, the corresponding parameter for the calcium store was implemented, reconnecting this component to the system. The selected parameters which negated electrical excitation are then introduced. To complete the system, the key variable connecting mechancial contraction to the rest of the model is included. By creating this set of parameters that included the CICR components, we are eventually able to study the model as a whole rather than individual components. This paper shows the design process of this set of parameters and then studies the influence of mechanical contraction 
on the rest of the model for simulations to an extended final time. This shows the possibility to study the influence of a particular problem parameter using the overall model.

This paper is organized as follows: Section 2 details the seven variable model used in this work. Section 3 specifies the numerical method used including its parallelization and implementation. Section 4 is divided into several subsections. Subsections 4.1-4.4 show the step-by-step implementation of the CICR components of the seven variable model. With the complete construction of the set of parameters, Subsection 4.5 presents a sample application using the new set of parameters. Section 5 collects the conclusions of the work. 


\section{Dynamics of a Cardiac Cell}

This section details the seven variable model used in this work. The seven variables of the model are calcium in the cytosol $c(\mathbf{x}, t)$, a florescent dye $b_{1}^{(c)}(\mathbf{x}, t)$, a contractile protein (troponin) $b_{2}^{(c)}(\mathbf{x}, t)$, a contractile force $b_{3}^{(c)}(\mathbf{x}, t)$, calcium in the sarcoplasmic reticulum (SR) $s(\mathbf{x}, t)$, voltage $V(\mathbf{x}, t)$, and the potassium gating function $n(\mathbf{x}, t)$. and are listed in Table 1 with their units and initial values. The evolution of the system is modeled by the system of seven time-dependent, coupled, non-linear reaction diffusion equations

$$
\begin{aligned}
& \frac{\partial c}{\partial t}= \nabla \cdot\left(D_{c} \nabla c\right)+R_{b_{1}}^{(c)}+R_{b_{2}}^{(c)}+\left(J_{C R U}+J_{\text {leak }}-J_{\text {pump }}\right) \\
&+\kappa J_{L C C}+\left(J_{m_{\text {leak }}}-J_{m_{\text {pump }}}\right), \\
& \frac{\partial b_{1}^{(c)}}{\partial t}= \nabla \cdot\left(D_{b_{1}^{(c)}} \nabla b_{1}^{(c)}\right)+R_{b_{1}}^{(c)}, \\
& \frac{\partial b_{2}^{(c)}}{\partial t}= \nabla \cdot\left(D_{b_{2}^{(c)}} \nabla b_{2}^{(c)}\right)+R_{b_{2}}^{(c)}, \\
& \frac{\partial b_{3}^{(c)}}{\partial t}=\nabla \cdot\left(D_{b_{3}^{(c)}} \nabla b_{3}^{(c)}\right)+R_{b_{3}}^{(c)}, \\
& \frac{\partial s}{\partial t}=\nabla \cdot\left(D_{s} \nabla s\right)-\gamma\left(J_{C R U}+J_{\text {leak }}-J_{\text {pump }}\right), \\
& \frac{\partial V}{\partial t}=\nabla \cdot\left(D_{v} \nabla V\right)+\tau_{v} \frac{1}{C}\left[I_{\text {app }}-g_{L}\left(V-V_{L}\right)-g_{K} n\left(V-V_{K}\right)\right. \\
&\left.-\frac{2 F}{S \tau_{f l u x}} J_{L C C}-\omega\left(J_{m_{\text {leak }}}-J_{m_{\text {pump }}}\right)\right], \\
& \frac{\partial n}{\partial t}= \nabla \cdot\left(D_{n} \nabla n\right)+\tau_{v} \lambda_{n} \cosh \left(\frac{V-V_{3}}{2 V_{4}}\right)\left(n_{\infty}(V)-n\right) .
\end{aligned}
$$

The domain in our model is a hexahedron

$$
\Omega=(-6.4,6.4) \times(-6.4,6.4) \times(-32.0,32.0)
$$

with units of $\mu \mathrm{m}$ with isotropic CRU distribution as sketched in Figure 1 (b) with a sample of three $z$-planes of $3 \times 3$ CRUs. A typical cell has on the order of $15 \times 15 \times 31=6,975$ calcium release units (CRUs) throughout the cell. A cell is reasonably modeled by the hexahedral shape elongated in the $z$-direction, since the focus of CICR research is on estimating correct physiological parameter values. The following provides details on each of the components of the model, the calcium signaling portion of the model, the electrical excitation that is connected to the calcium signaling in both the feedforward and feedback directions represented by link (1) and link (2) in Figure 1 (a), and the mechanical contraction component that is also connected to the calcium signaling in both the feedback and feedforward directions represented by links (3) and (4) in Figure 1 (a).

Tables 2 and 3 contain the parameters in the PDEs of the calcium system with their values (if fixed) and units; some of the values are varied in the experiments in Section 4 . The coefficients $D_{c}, D_{b_{i}^{(c)}}$, and $D_{s}$ are the diffusivity matrices for $\mathrm{Ca}^{2+}$ in the cytosol, buffer species $i$ in the cytosol, and $\mathrm{Ca}^{2+}$ in the SR, respectively.

The calcium signaling portion of the model consists of the equations (1)-(5). The reaction terms $R_{b_{i}}^{(c)}$ describe the reactions between calcium and the buffer species. 


\begin{tabular}{lll}
\hline Variable & Definition & Initial value and units \\
\hline $\mathbf{x}$ & spatial position variable $(x, y, z)$ & $\mu \mathrm{m}$ \\
$t$ & time variable & $\mathrm{ms}$ \\
\hline$c(\mathbf{x}, t)$ & calcium in the cytosol & $c_{0}=0.1 \mu \mathrm{M}$ \\
$b_{1}^{(c)}(\mathbf{x}, t)$ & free flourescent dye in the cytosol & $45.918 \mu \mathrm{M}$ \\
$b_{2}^{(c)}(\mathbf{x}, t)$ & free troponin in the cytosol & $111.818 \mu \mathrm{M}$ \\
$b_{3}^{(c)}(\mathbf{x}, t)$ & inactive actin-myosin cross-bridges $[\mathrm{X}]$ & $145.20 \mu \mathrm{M}$ \\
$s(\mathbf{x}, t)$ & calcium in the SR & $s_{0}=10,000 \mu \mathrm{M}$ \\
$V(\mathbf{x}, t)$ & membrane potential (voltage) & $-50 \mathrm{mV}$ \\
$n(\mathbf{x}, t)$ & fraction of open potassium channels & 0.1 \\
\hline
\end{tabular}

TABLE 1. Independent and dependent variables of the seven variable model and their initial conditions. The concentration unit M is shorthand for mol/L (moles per liter).

They are the connections between (1)-(4). More precisely, we have

$$
R_{b_{1}}^{(c)}=-k_{b_{1}^{(c)}}^{+} c b_{1}^{(c)}+k_{b_{1}^{(c)}}^{-}\left(b_{1, \text { total }}^{(c)}-b_{1}^{(c)}\right),
$$

for reaction between cytosol calcium and the flourescent dye.

When troponin binds to $\mathrm{Ca}^{2+}$, the protein as a whole changes shape: this not only allow actin-myosin cross-bridges to form, but also traps the calcium in its connection to the troponin so that the disassociation rate of troponin binding to $\mathrm{Ca}^{2+}$ decreases dramatically. To account for this, the shortening factor $\varepsilon$ describes how the separation of troponin and calcium has been physically, but not chemically, impaired. When there are open CRUs that resultantly release calcium in the cytosol, this chain of changes of variables increases in severity. However, after $t=5 \mathrm{~ms}$ of being open, the CRUs close and remain in a resting state for $t=100 \mathrm{~ms}$. During this period, the unbinding of troponin and calcium increase to the intial rate of unbinding and myosin cross-bridges become inactive. This results in these terms returning to their orignal states. This cycle is continuous triggered and reset by the calcium waves produced by a cardiomyocyte. Note, again, that $R_{b_{2}}^{(c)}$ remains a function of cytosol calcium concentration $c(\mathbf{x}, t)$ by its equation

$$
R_{b_{2}}^{(c)}=-k_{b_{2}^{(c)}}^{+} c b_{2}^{(c)}+\left(\frac{k_{b_{2}^{(c)}}^{-}}{\varepsilon}\right)\left(b_{2, \text { total }}^{(c)}-b_{2}^{(c)}\right)
$$

with

$$
\varepsilon=\exp \left(F_{\max } k_{s}\left(\frac{b_{3, \text { total }}^{(c)}-b_{3}^{(c)}-[X B]_{0}}{b_{3, \text { total }}^{(c)}-[X B]_{0}}\right)\right)
$$

and $[X B]_{0}=b_{3, \text { total }}^{(c)}-b_{3}^{(c)}(\mathbf{x}, 0)$. This shortening factor $\varepsilon$ links (3) and (4) in Figure 1 (a). It is important to note that the disassociation rate of troponin binding to $\mathrm{Ca}^{2+}$ is highest when the calcium concentration in the cytosol is lowest.

The shortening factor refers back to the concentration of $b_{3}^{(c)}(\mathbf{x}, t)$, the actinmyosin cross-bridges, and to the force that their linkage generates. It is scaled by the maximum possible contractile force $F_{\max }$, the actin stiffness $k_{s}$, and the 


\begin{tabular}{|c|c|c|}
\hline Variable & Definition & Values/Units \\
\hline$D_{c}$ & diffusivity matrix for $c(\mathbf{x}, t)$ & $\operatorname{diag}(0.15,0.15,0.3) \mu \mathrm{m}^{2} / \mathrm{ms}$ \\
\hline$D_{b_{1}^{(c)}}$ & diffusivity matrix for $b_{1}^{(c)}$ & $\operatorname{diag}(0.01,0.01,0.02) \mu \mathrm{m}^{2} / \mathrm{ms}$ \\
\hline$D_{b_{2}^{(c)}}$ & diffusivity matrix for $b_{2}^{(c)}$ & $\operatorname{diag}(0.00,0.00,0.00) \mu \mathrm{m}^{2} / \mathrm{ms}$ \\
\hline$D_{b_{3}^{(c)}}^{2}$ & diffusivity matrix for $b_{3}^{(c)}$ & $\operatorname{diag}(0.00,0.00,0.00) \mu \mathrm{m}^{2} / \mathrm{ms}$ \\
\hline$D_{s}{ }^{3}$ & diffusivity matrix for $s(\mathbf{x}, t)$ & $\operatorname{diag}(0.78,0.78,0.78) \mu \mathrm{m}^{2} / \mathrm{ms}$ \\
\hline$R_{b_{i}}^{(c)}$ & reactions of cytosol $\mathrm{Ca}^{2+}$ with buffers & $\mu \mathrm{M} / \mathrm{ms}$ \\
\hline$k_{b_{1}^{(c)}}^{+}$ & forward reaction coefficient for $b_{1}^{(c)}$ & $0.080(\mu \mathrm{M} \mathrm{ms})^{-1}$ \\
\hline$k_{b_{2}^{(c)}}^{+}$ & forward reaction coefficient for $b_{2}^{(c)}$ & $0.100(\mu \mathrm{M} \mathrm{ms})^{-1}$ \\
\hline$k_{b_{3}^{(c)}}^{+}$ & forward reaction coefficient for $b_{3}^{(c)}$ & $0.040 \mathrm{~ms}^{-1}$ \\
\hline$k_{b_{1}^{(c)}}^{-}$ & reverse reaction coefficient for $b_{1}^{(c)}$ & $0.090 \mathrm{~ms}^{-1}$ \\
\hline$k_{b_{2}^{(c)}}^{-}$ & reverse reaction coefficient for $b_{2}^{(c)}$ & $0.100 \mathrm{~ms}^{-1}$ \\
\hline$k_{b_{3}^{(c)}}^{-}$ & reverse reaction coefficient for $b_{3}^{(c)}$ & $0.010 \mathrm{~ms}^{-1}$ \\
\hline$b_{1, \text { total }}^{(c)}$ & total amount of $b_{1}^{(c)}$ in the cytosol & $50 \mu \mathrm{M}$ \\
\hline$b_{2, \text { total }}^{(c)}$ & total amount of $b_{2}^{(c)}$ in the cytosol & $123 \mu \mathrm{M}$ \\
\hline$b_{3, \text { total }}^{(c)}$ & total amount of $b_{3}^{(c)}$ in the cytosol & $150 \mu \mathrm{M}$ \\
\hline$\gamma$ & ratio of volume of cytosol to SR & 14 \\
\hline$J_{\text {leak }}$ & calcium leak from SR & $0.3209684 \mu \mathrm{M} / \mathrm{ms}$ \\
\hline$J_{\text {pump }}$ & calcium transfer from cytosol to SR & $\mu \mathrm{M} / \mathrm{ms}$ \\
\hline$V_{\text {pump }}$ & maximum pump rate & $4 \mu \mathrm{M} / \mathrm{ms}$ \\
\hline$K_{\text {pump }}$ & pump sensitivity to $\mathrm{Ca}^{2+}$ & $0.184 \mu \mathrm{M}$ \\
\hline$n_{\text {pump }}$ & Hill coefficient for pump function & 4.0 \\
\hline$J_{C R U}$ & calcium flux from SR to cytosol via CRUs & $\mu \mathrm{M} / \mathrm{ms}$ \\
\hline & gating function for $J_{C R U}$ & 0 or 1 \\
\hline$J_{\text {prob }}$ & probability of CRU opening & 0 to 1 \\
\hline $\mathbf{x}_{s}$ & three-dimensional vector for CRU location & $\mu \mathrm{m}$ \\
\hline$\Delta x_{s}$ & CRU spacings in $x$ & $0.8 \mu \mathrm{m}$ \\
\hline$\Delta y_{s}$ & CRU spacings in $y$ & $0.8 \mu \mathrm{m}$ \\
\hline$\Delta z_{s}$ & CRU spacings in $z$ & $2.0 \mu \mathrm{m}$ \\
\hline & maximum rate of release & $110 \mu \mathrm{M} \mu \mathrm{m}^{3} / \mathrm{ms}$ \\
\hline$\delta(\mathbf{x}-\hat{\mathbf{x}})$ & Dirac delta distribution & $1 / \mu \mathrm{m}^{3}$ \\
\hline$u_{\text {rand }}$ & uniformly distributed random variable & 0 to 1 \\
\hline$P_{\max }$ & maximum probability for release & 0.3 \\
\hline$K_{\text {prob }_{c}}$ & sensitivity of CRU to cytosol calcium & $2 \mu \mathrm{M}$ \\
\hline$n_{\text {prob }_{c}}$ & Hill coefficient for probability function & 4 \\
\hline$K_{\text {probs }}$ & sensitivity of CRU to $\mathrm{SR}$ calcium & $550 \mu \mathrm{M}$ \\
\hline$n_{\text {probs }_{s}}$ & Hill coefficient for probability function & 4 \\
\hline
\end{tabular}

TABLE 2. Parameters for calcium signaling.

proportion of active to inactive actin-myosin cross-bridges. As the proportion of active actin-myosin cross-bridges increases due to an increase in bounded troponin, the value of $b_{3, \text { total }}^{(c)}-b_{3}^{(c)}-[X B]_{0}$ increases. This results in a larger value of $\varepsilon$. As $\varepsilon$ becomes larger, the rate at which the bounded troponin unbinds slows down. As described earlier, the lack of calcium provided by CRU resting periods results in a reset in this mechanism.

The contractile proteins in question, though considered as a single species, are the combination of actin and myosin when linked via cross-bridges. This linkage 


\begin{tabular}{|c|c|c|}
\hline Variable & Definition & Values/Units \\
\hline$D_{v}$ & diffusivity matrix for $V(\mathbf{x}, t)$ & $\operatorname{diag}(100,100,100) \mu \mathrm{m}^{2} / \mathrm{ms}$ \\
\hline$D_{n}$ & diffusivity matrix for $n(\mathbf{x}, t)$ & $\operatorname{diag}(0,0,0) \mu \mathrm{m}^{2} / \mathrm{ms}$ \\
\hline$\tau_{v}$ & scaling factor in voltage equation & $0.1 \mu \mathrm{M} \mu \mathrm{m}^{3} / \mathrm{ms}$ \\
\hline$\tau_{f l u x}$ & scaling factor in $J_{L C C}$ term & 0.1 \\
\hline$V_{1}$ & potential at which $m_{\infty}=0.5$ & $-1.0 \mathrm{mV}$ \\
\hline$V_{2}$ & reciprocal of slope of voltage dependence of $m_{\infty}$ & $15.0 \mathrm{mV}$ \\
\hline$V_{3}$ & potential at which $n_{\infty}=0.5$ & $10.0 \mathrm{mV}$ \\
\hline$V_{4}$ & reciprocal of slope of voltage dependence of $n_{\infty}$ & $14.5 \mathrm{mV}$ \\
\hline$V_{L}$ & equilibrium potential for leak conductance & $-50 \mathrm{mV}$ \\
\hline$V_{C a}$ & equilibrium potential for $\mathrm{Ca}^{2+}$ conductance & $100 \mathrm{mV}$ \\
\hline$V_{K}$ & equilibrium potential for $\mathrm{K}^{+}$conductance & $-70 \mathrm{mV}$ \\
\hline$C$ & membrane capacitance & $20 \mu \mathrm{F} / \mathrm{cm}^{2}$ \\
\hline$I_{a p p}$ & applied current & $50 \mu \mathrm{A} / \mathrm{cm}^{2}$ \\
\hline$g_{L}$ & maximum/instantaneous conductance for leak & $2 \mathrm{mmho} / \mathrm{cm}^{2}$ \\
\hline$g_{C a}$ & maximum/instantaneous conductance for $\mathrm{Ca}^{2+}$ & $4 \mathrm{mmho} / \mathrm{cm}^{2}$ \\
\hline$g_{K}$ & maximum/instantaneous conductance for $\mathrm{K}^{+}$ & $8 \mathrm{mmho} / \mathrm{cm}^{2}$ \\
\hline$m_{\infty}$ & fraction of open calcium channels at steady state & 0 to 1 \\
\hline$n_{\infty}$ & fraction of open potassium channels at steady state & 1 \\
\hline$\lambda_{n}$ & maximum rate constant for opening of $\mathrm{K}^{+}$channels & $0.1 \mathrm{~ms}^{-1}$ \\
\hline$J_{L C C}$ & influx of calcium via L-type calcium channels & $\mu \mathrm{M} / \mathrm{ms}$ \\
\hline$S$ & surface area of the cell & $3604.48 \mu \mathrm{m}^{2}$ \\
\hline$F$ & Faraday constant & $95484.56 \mathrm{C} / \mathrm{mol}$ \\
\hline$\kappa$ & scaling factor of $J_{L C C}$ & 0.1 \\
\hline$\omega$ & feedback strength (scaling factor) for $\mathrm{Ca}^{2+}$ efflux & $100 \mu \mathrm{A} \mathrm{ms} / \mu \mathrm{M} \mathrm{cm}^{2}$ \\
\hline$J_{m_{l e a k}}$ & leak of calcium via L-type calcium channels & $0.1739493 \mu \mathrm{M} / \mathrm{ms}$ \\
\hline$J_{m_{p u m p}}$ & pump of calcium via L-type calcium channels & $\mu \mathrm{M} / \mathrm{ms}$ \\
\hline$V_{m_{p u m p}}$ & maximum pump rate & $0.3 \mu \mathrm{M} / \mathrm{ms}$ \\
\hline$n_{m_{p u m p}}$ & membrane pump Hill coefficient & 4 \\
\hline$K_{m \text { pump }}$ & membrane pump $\mathrm{Ca}^{2}+$ sensitivity & 0.18 \\
\hline$[X B]_{0}$ & initial concentration of active cross-bridges & $142.6805 \mu \mathrm{M}$ \\
\hline$\varepsilon$ & shortening factor & 0 to 1 \\
\hline$F_{\max }$ & maximum force by actin-myosin crossbridges & $1 \mu \mathrm{N}$ \\
\hline$k_{s}$ & stiffness of actin filament & $0.025 \mathrm{~N} / \mathrm{m}$ \\
\hline
\end{tabular}

TABLE 3. Parameters for electrical excitation and mechanical contraction with base units of mho $=\left(\mathrm{s}^{3} \mathrm{~A}^{2}\right) /\left(\mathrm{kg} \mathrm{m}^{2}\right)$ and $\mathrm{F}=$ $\left(\mathrm{s}^{4} \mathrm{~A}^{2}\right) /\left(\mathrm{kg} \mathrm{m}^{2}\right)$.

is made possible by $\mathrm{Ca}^{2+}$ binding to troponin, the cytosol buffer species $b_{2}^{(c)}(\mathbf{x}, t)$ : it is this binding that allows the actin-myosin cross-bridges to form. The cytosol species, $b_{3}^{(c)}(\mathbf{x}, t)$, describes these actin-myosin cross-bridges and constructs a third cytosol reaction term

$$
R_{b_{3}}^{(c)}=-k_{b_{3}^{(c)}}^{+}\left(\frac{b_{2, \text { total }}^{(c)}-b_{2}^{(c)}}{b_{2, \text { total }}^{(c)}}\right)^{2} b_{3}^{(c)}+k_{b_{3}^{(c)}}^{-}\left(b_{3, \text { total }}^{(c)}-b_{3}^{(c)}\right) .
$$

Notice that this is not the same as the generic pattern for buffer species reaction terms from the initial model. There is no immediately clear dependence on cytosolic calcium $c(\mathbf{x}, t)$. However, while $c(\mathbf{x}, t)$ is not explicitly included, it is present in the proportion involving troponin, $b_{2}^{(c)}(\mathbf{x}, t)$, which itself depends explicitly on cytosol calcium levels; $R_{b_{3}}^{(c)}$, like the other two reaction equations, does in fact depend on 
the cytosol calcium concentration. Unlike the other two reaction equations, $R_{b_{3}}^{(c)}$ indirectly impacts calcium levels.

These two reaction terms (10) and (12) connect the three components of our model. The calcium signaling is linked to the pseudo-mechanical contraction by the cross-bridge term, and the pseudo-mechanical contraction is in turn connected to the calcium signaling through the inclusion of the cytosol calcium concentration in the modified reaction equation for troponin. Thus all links (1), (2), (3), and (4) in Figure 1 (a) are established, and thus the three systems of the model are fully linked.

Note that in the reaction terms $b_{2}{ }^{(c)}$ and $b_{3}{ }^{(c)}$ are the amount of unbound buffer known as "free" buffer. The constant $b_{i, t o t a l}^{(c)}$ denotes the total bound and unbound calcium thus leaving the difference seen in (9) to be the bound calcium. Since the model uses no-flux boundary conditions, no buffer species escapes or enters the cell, thus we only need to track the "free" buffer species and use $b_{i, t o t a l}^{(c)}-b_{i}{ }^{(c)}$ for the bound species.

The flux terms $J_{C R U}, J_{\text {leak }}$, and $J_{\text {pump }}$ in (1) describe the calcium induced release of $\mathrm{Ca}^{2+}$ into the cytosol from the $\mathrm{SR}$, the continuous leak of $\mathrm{Ca}^{2+}$ into the cytosol from the SR, and the pumping of $\mathrm{Ca}^{2+}$ back into the SR from the cytosol. The terms $J_{L C C}, J_{m_{\text {leak }}}$, and $J_{m_{\text {pump }}}$ describe the fluxes of calcium into and out of the cell via the plasma membrane. The coupling between (1) and (5) is achieved by the three flux terms shared by both.

More precisely, $J_{L C C}, J_{m_{\text {leak }}}$, and $J_{m_{\text {pump }}}$ in (1) describe the fluxes of calcium into and out of the cell via the plasma membrane. $J_{\text {pump }}$ replenishes the calcium stores in the SR; it increases SR calcium concentration by decreasing cytosol calcium concentration. $J_{\text {leak }}$ is a continuous leakage of those SR calcium stores into the cytosol; it increases cytosol concentration by decreasing SR calcium concentration. The pump term

$$
J_{\text {pump }}(c)=V_{\text {pump }}\left(\frac{c^{n_{\text {pump }}}}{K_{\text {pump }}^{n_{\text {pump }}}+c^{n_{\text {pump }}}}\right)
$$

is thus a function of cytosol calcium $c(\mathbf{x}, t)$. The leak term $J_{\text {leak }}$ is a constant defined by

$$
J_{\text {leak }}=J_{\text {pump }}\left(c_{0}\right)
$$

which balances $J_{\text {pump }}(c)$ at basal level $c_{0}=0.1 \mu \mathrm{M}$ of cytosol calcium. The pump term $J_{\text {pump }}$, a function of cytosolic calcium $c(\mathbf{x}, t)$, consists of the maximum pump velocity $V_{\text {pump }}$ multiplied against the relationship between $c(\mathbf{x}, t)$ and the pump sensitivity $K_{\text {pump }}$; the exponent $n_{\text {pump }}$ refers to the Hill coefficient (quantifying the degree of cooperative binding) for the pump function. This has the practical effect of multiplying the maximum possible pump velocity against a number between 0 and 1, exclusive. $J_{\text {leak }}$, which continuously leaks calcium into the cytosol from the SR, is simply $J_{\text {pump }}$ evaluated at the basal cytosolic calcium concentration $c_{0}=0.1 \mu M$. As noted, $J_{\text {pump }}$ has two roles, namely to balance $J_{\text {leak }}$ in the absence of sparking, but also to balance $J_{C R U}$ under conditions of active calcium release.

The term $J_{C R U}$ in (1) is the $\mathrm{Ca}^{2+}$ flux into the cytosol from the SR via each individual point source at which a CRU has been assigned. The effect of all CRUs 
is modeled as a superposition such that

$$
J_{C R U}(c, s, \mathbf{x}, t)=\sum_{\hat{\mathbf{x}} \in \Omega_{s}} \hat{\sigma} \frac{s(\mathbf{x}, t)}{s_{0}} \mathcal{O}(c, s) \delta(\mathbf{x}-\hat{\mathbf{x}})
$$

with

$$
\mathcal{O}(c, s)= \begin{cases}1 & \text { if } u_{\text {rand }} \leq J_{\text {prob }} \\ 0 & \text { if } u_{\text {rand }}>J_{\text {prob }}\end{cases}
$$

where

$$
J_{\text {prob }}(c, s)=P_{\max }\left(\frac{c^{n_{\text {prob }}}}{K_{\text {prob }_{c}}^{n_{\text {probc }}}+c^{n_{\text {probc }}}}\right)\left(\frac{s^{n_{\text {probs }}}}{K_{\text {prob }}^{n_{\text {probs }}}+s^{n_{\text {probs }}}}\right) .
$$

The effect of each CRU is modeled here as a product of three terms: (i) Similarly to how in $J_{\text {pump }}$ the maximum pump rate is scaled against the concentration of available cytosol calcium, the maximum pump rate is scaled against the concentration of available cytosol calicum, the maximum rate of $\mathrm{Ca}^{2+}$ release $\hat{\sigma}$ is scaled here against the ratio of calcium concentration in the SR. (ii) Following the same pattern a maximum value multiplied against some scaling proportion between 0 and 1 the gating function $\mathcal{O}(c, s)$ has the practical effect of "budgeting" the calcium SR stores such that when the stores are low, the given CRU becomes much less likely to open; each CRU is assigned a uniformly distributed random value $u_{\text {rand }}$, which is compared to the single value returned by the CRU opening probability $J_{\text {prob }}$ to determine whether or not the given CRU will open. (iii) The Dirac delta distribution $\delta(\mathbf{x}-\hat{\mathbf{x}})$ models each CRU as a point source for calcium release, which is defined by requiring $\delta(\mathbf{x}-\hat{\mathbf{x}})=0$ for all $\mathbf{x} \neq \hat{\mathbf{x}}$ and $\int_{\mathbb{R}^{3}} \psi(\mathbf{x}) \delta(\mathbf{x}-\hat{\mathbf{x}}) d \mathbf{x}=\psi(\hat{\mathbf{x}})$ for any continuous function $\psi(\mathbf{x})$.

The linking between calcium signaling and electrical excitation consists of (6)(7). The membrane potential of the cell depends on both the cytosol calcium ion concentration and also on the cytosol potassium ion $\left(\mathrm{K}^{+}\right)$concentration $\left[\mathrm{BHJ}^{+} 12\right.$, ML81]. In our model, the $\omega$ term in (6) quantifies a dependence of $V$ on $c$ to complete the coupling from the chemical to the electrical systems in link (2) in Figure 1 (a) $\left[\mathrm{ABM}^{+} 16\right]$, after $c$ in (1) already contains several terms that depend on $V$ to implement link (1) in Figure 1 (a). The $\mathrm{Ca}^{2+}$ conductance is much faster than the $\mathrm{K}^{+}$conductance, so the calcium conductance can be approximated as $m_{\infty}$ or instantaneously steady-state at all times; the potassium conductance requires a separate description in (7). Needed parameter functions in (6)-(7) are

$$
\begin{aligned}
m_{\infty}(V) & =\frac{1}{2}\left(1+\tanh \left(\frac{V-V_{1}}{V_{2}}\right)\right), \\
n_{\infty}(V) & =\frac{1}{2}\left(1+\tanh \left(\frac{V-V_{3}}{V_{4}}\right)\right) .
\end{aligned}
$$

The connection between (1) and (6), link (1) in Figure 1 (a), the link from the electrical system to the calcium system, comes through

$$
J_{L C C}=\frac{\tau_{f l u x}}{2 F} S g_{C a} m_{\infty}(V)\left(V-V_{C a}\right),
$$

the only calcium flux term to involve voltage. Note the parameter $\kappa$ in (1), which is an external scaling factor for $J_{L C C}$ rather than an intrinsic physiological component; if the value of $\kappa$ is set to 0 , the connection, link (1) in Figure 1 (a), is effectively switched off and the calcium dynamics are then modeled as though voltage were not 
involved. The surface area, $S$, of the cell is included in light of the fact that $J_{L C C}$ describes the influx of calcium through L-type calcium channels (LCCs), which are present in the enclosing plasma membrane of the cell: the surface area of the cell is the surface area of the membrane.

We model the effect of the cytosol calcium concentration on the voltage by treating the calcium efflux term $\left(J_{m_{\text {pump }}}-J_{m_{\text {leak }}}\right)$ as equivalent to the sodiumcalcium exchanger current: we are thus able to describe the current generated by the sodium-calcium exchange as a function of simple calcium loss.

The individual components of the calcium efflux term are near-duplicates in form of the earlier $J_{\text {pump }}$ and $J_{\text {leak }}$ functions in (13) and (14), respectively. As $J_{\text {pump }}$ described the removal of calcium from the cytosol and its transfer into SR stores,

$$
J_{m_{\text {pump }}}(c)=V_{m_{\text {pump }}}\left(\frac{c^{n_{m_{\text {pump }}}}}{K_{m_{\text {pump }}}^{n_{m_{\text {pump }}}}+c^{n_{m_{\text {pump }}}}}\right)
$$

describes the removal of calcium from the cytosol and its transfer to outside the cell across the membrane. The leak term $J_{\text {leak }}$ described a gradual leak of calcium into the cytosol from the SR, while $J_{C R U}$ described an abrupt, high-concentration (high relative to the leak) release of calcium into the cytosol from the SR. Similarly,

$$
J_{m_{\text {leak }}}=J_{m_{\text {pump }}}\left(c_{0}\right)
$$

describes a gradual leak of calcium into the cytosol from outside the cell via the plasma membrane, while $J_{L C C}$ describes a sudden spike of calcium release into the cytosol via the LCCs.

The seven variable model presented in this section reduces to the three variable model in the original sources [IMBW01, IWB01] by the following choies: setting $\gamma$ $=0, \tau_{v}=0, \omega=0, \kappa=0$, and $F_{\max }=0$. With these parameters set to 0 , linkages (1), (2), (3), and (4) in Figure 1 (a) are all cut off. 


\section{Numerical Method}

In order to do calculations for the CICR model, we need to solve a system of time-dependent parabolic partial differential equations (PDEs). These PDEs are coupled by several non-linear reaction and source terms. The current simulations use $n_{s}=7$ physiological variables. The domain in our model is a hexahedron with isotropic CRU distribution as shown in Figure 1 (b). A typical cell has on the order of $15 \times 15 \times 31=6,975$ calcium release units (CRUs) throughout the cell. A cell is reasonably modeled by the hexahedral shape elongated in the $z$-direction, since the focus of CICR research is on estimating correct physiological parameter values. Taking a method of lines (MOL) approach to spatially discretize this model, we use the finite volume method (FVM) as the spatial discretization to ensure mass conservation on the discrete level and also to accommodate advection terms in the future, with $N=\left(N_{x}+1\right)\left(N_{y}+1\right)\left(N_{z}+1\right)$ control volumes. Applying this to the case of the $n_{s}$ PDEs results in a large system of ordinary differential equations (ODEs). A MOL discretization of a diffusion-reaction equations with second-order spatial derivatives results in a stiff ODE system. The time step size restrictions, due to the CFL condition, are considered too severe to allow for explicit time-stepping methods. This necessitates the use of a sophisticated ODE solver such as the family of implicit numerical differentiation formulas (NDF $k$ ). We use Newton's method as the non-linear solver, and at each Newton step we use the biconjugate gradient stabilized method (BiCGSTAB) or other Krylov subspace methods as the linear solver. Complete details of the numerical method can be found in $\left[\mathrm{SHK}^{+} 15\right]$.

\begin{tabular}{rrrrrr}
\hline Mesh resolution & $N$ & DOF $n_{e q}$ & time steps & \multicolumn{2}{c}{ memory $(\mathrm{GB})$} \\
$N_{x} \times N_{y} \times N_{z}$ & & & & predicted & observed \\
\hline $32 \times 32 \times 128$ & 140,481 & 983,367 & 2667 & 0.125 & $<1$ \\
$64 \times 64 \times 256$ & $1,085,825$ & $7,600,775$ & 3295 & 0.963 & 1.093 \\
$128 \times 128 \times 512$ & $8,536,833$ & $59,757,831$ & 3867 & 7.569 & 8.476 \\
$256 \times 256 \times 1024$ & $67,700,225$ & $473,901,575$ & 4470 & 60.024 & 67.103 \\
\hline
\end{tabular}

TABLE 4 . Sizing study with $n_{s}=7$ variables using double precision arithmetic, listing the mesh resolution $N_{x} \times N_{y} \times N_{z}$, the number of control volumes $N=\left(N_{x}+1\right)\left(N_{y}+1\right)\left(N_{z}+1\right)$, the number of degrees of freedom (DOF) $n_{e q}=n_{s} N$, the number of time steps taken by the ODE solver up to final time $t_{\text {fin }}=100 \mathrm{~ms}$, and the predicted and observed memory usage in GB for a one-process run.

While the form of the PDEs in (1)-(7) is customary, the thousands of point sources at the calcium release units (CRUs) in the forcing terms imply that standard codes have difficulty. This explains why our research centered around creating a special-purpose code [Gob08, HGI04, SGTK12, $\mathrm{SHK}^{+}$15]. Additionally, we leverage the regular 3-D shape of the domain to program all methods in matrix-free form. Table 4 demonstrates thus, how despite fully implicit time-stepping, even relatively fine meshes use only modest amounts of memory. This provides the key benefit now for the complete complex model with complete sophisticated numerical method stack, even with 7 or 8 variables, to fit into the high-performance memory of a KNL or a GPU. The table also shows how large the number of degrees of freedom (DOF) 
is, that is, the number of variables that have to be computed at every time step. With even modest meshes, there are millions of unknowns, and possibly hundreds of millions for fine meshes. This characterizes the numerical problem that needs to be tackled to resolve the large number of thousands of calcium release units (CRUs) in a cell.

The implementation of this model is done in C using MPI and OpenMP to parallelize computations. Parallelization is accomplished through block-distribution all large arrays to all MPI processes. We split of the mesh in the $z$-direction with one subdomain on each of the parallel processes. MPI commands such as MPI_Isend and MPI_Irecv, which are non-blocking point-to-point communication commands, send messages between neighboring processes. The collective command MPI_Allreduce is used for the computation of scalar products and norms. 


\section{Results}

This section shows the results of simulations for the seven variable model detailed in Section 2 with variables aand their values at the initial time $t=0 \mathrm{~ms}$ specified in Table 1. The following subsections show physiological output that is possible through long-time computational simulations to a final time that is large enough to include several calcium waves. Tables 2 and 3 specify all model parameters, their names, and their values or ranges with units, except certain parameters that are varied in this section. Table 5 specifies for each of the subsections, what values are used. In Tables 2 and 3, the values of the variables are selected from Table 5. Subsection 4.1 connects to the original modeling in [IMBW01, IWB01] by performing simulations with parameters chosen such that the seven variable model reduces to the original three variable model used in these papers. The following Subsections 4.2, 4.3, and 4.4 successively vary parameters shown in Table 5 , in addition to the parameter changes made in the previous subsections. This approach allows for the construction of the entire model by adding new components on top of previous ones. With the entire model pieced together, we can test the model as a whole. That is the purpose of Subsection 4.5 that briefly exhibits the power of the fully implemented model by showing the impact of $F_{\max }=1$ and $F_{\max }=100$ on the entire system for $t=5,000 \mathrm{~ms}$, a longer duration of time. These results show the power of a fully functioning system and motivate further studies using this model.

\begin{tabular}{lrrrrr}
\hline Parameter & Base case & SR store & Voltage system & Mechanical system \\
& Sec. 4.1 & Sec. 4.2 & Sec. 4.3 & Sec. 4.4 & Sec. 4.5 \\
\hline$\gamma$ & 0.0 & 0.01 to 1000 & 14.0 & 14.0 & 14.0 \\
\hline$\tau_{v}$ & 0.0 & 0.0 & 0.1 & 0.1 & 0.1 \\
$V_{m_{\text {pump }}}$ & 0.0 & 0.0 & 0.001 to 1 & 0.3 & 0.3 \\
$\omega$ & 0.0 & 0.0 & 0.1 to 1000 & 100.0 & 100.0 \\
$\kappa$ & 0.0 & 0.0 & 0.001 to 10 & 0.1 & 0.1 \\
\hline$F_{\max }$ & 0.0 & 0.0 & 0.0 & 0.001 to 100 & 1,100 \\
\hline$t_{\text {fin }}$ & 1000 & 1000 & 1000 & 1000 & 5000 \\
\hline
\end{tabular}

TABle 5. Parameter differences between cases in sections. 
4.1. Base Case. The seven variable model in (1)-(7) reduces to the three variable model in the original sources [IMBW01, IWB01] by the following choices: setting $\kappa=0, F_{\max }=0$, and $\gamma=0$. When looking at the seven equations, changing these parameters to zero is most appropriate as they result in removing the calcium store in the Sarcoplasmic Reticulum (SR) and the effects of mechanical contraction.

The final addition of the three variable model, electrical excitation, can be absolutely removed by setting ( 6 and 7 ) to zero. Changing $\tau_{v}=0$ acheives this. These three components are only unique to the seven variable model. To confirm that the first equation, (1), acts identically to the first equation in [IMBW01], we must be absolutely sure that $J_{m_{\text {leak }}}-J_{m_{\text {pump }}}=0$. To do this, we change $V_{m_{\text {pump }}}=0$ and $\omega=0$, resulting in (21) negating to zero and both sides of (22) equaling zero. With these additional changes, the seven variable model functions identically to the three variable model in the original sources [IMBW01, IWB01].

The first set of plots, in Figure 2, display the locations of open calcium release units by a dot. The more dark dots are visible, the more CRUs are open at that specific time. More specifically, since one open CRU tends to promote neighboring CRUs to open through the diffusion of calcium, a clustering of dots indicates a region of several open CRUs. Note that we start with initial conditions in Table 5 for which there are no open CRUs and the right-hand sides of all equations in (1)(7) are zero. Thus, these simulations represent a study in spontaneous sparking, in which some CRUs happen to open according to the probabilistic model in (15)(17). This model embodies the effect of a higher concentration of cytosol calcium increasing the probability for a CRU to open in (17). The result of this spontaneous self-initiation is seen in the first sub-plot in Figure 2, where a number of CRUs in one region of the cell have opened by $t=100 \mathrm{~ms}$. The study of self-initiation was the original purpose of this model of calcium induced calcium releases [IMBW01, IWB01] and only required modest final times. The extension of the simulations to larger final times allows to study if the opening and closing of CRUs self-organizes into a sustained wave. This is seen in the following sub-plots in Figure 2. The model requires CRUs to close for $100 \mathrm{~ms}$ after opening for $5 \mathrm{~ms}$; thus the sub-plots indicate that the original cluster of open CRUs travels in both directions through the elongated direction of the cell and roughly repeats every $100 \mathrm{~ms}$. Movies of the full results including intervening times confirm this behavior.

Figure 3 shows a collection of isosurface plots for calcium concentration in the cytosol. An isosurface plot displays the surface in the three-dimensional cell, where the species concentration is equal to a critical value, stated in the caption of the figure, here $65 \mu \mathrm{M}$ for the concentration of cytosol calcium $c(\mathbf{x}, t)$. Blue represents when the species' concentration equals $65 \mu \mathrm{M}$. For densities lower than $65 \mu \mathrm{M}$, there are no markings. More extreme concentrations of each species are indicated with the spectrum from yellow to red, where darker hues are more intense in density. As we can see from the sub-plots in Figure 2, we have an original cluster in the middle at $t=100 \mathrm{~ms}$ that diffuses towards the ends of the cell and repeats on a $100 \mathrm{~ms}$ cycle. Due to the connection of the calcium in the cytosol with open CRUs through the effect of calcium induced calcium release, calcium concentrations in the cytosol corresponds to locations of open CRUs.

This physiological concept is shown with each sub-plot in Figure 3 where the species' concentration colors are in agreement with the amount of open CRUs in Figure 2 at that time. 
The concentrations of $b_{1}^{(c)}(\mathbf{x}, t)$ and $b_{2}^{(c)}(\mathbf{x}, t)$ have the same charactersitics as the calcium levels in the cytosol since they are all influenced in the same way, therefore, we do not show them. The remaining isosurface plots for the base case are not included because each of the additional species concentration remain constant. Resultantly, each subplot would remain uneventful. As the CICR components are added to the base case in the following subsections, the respective isosurface plots will also be included. 

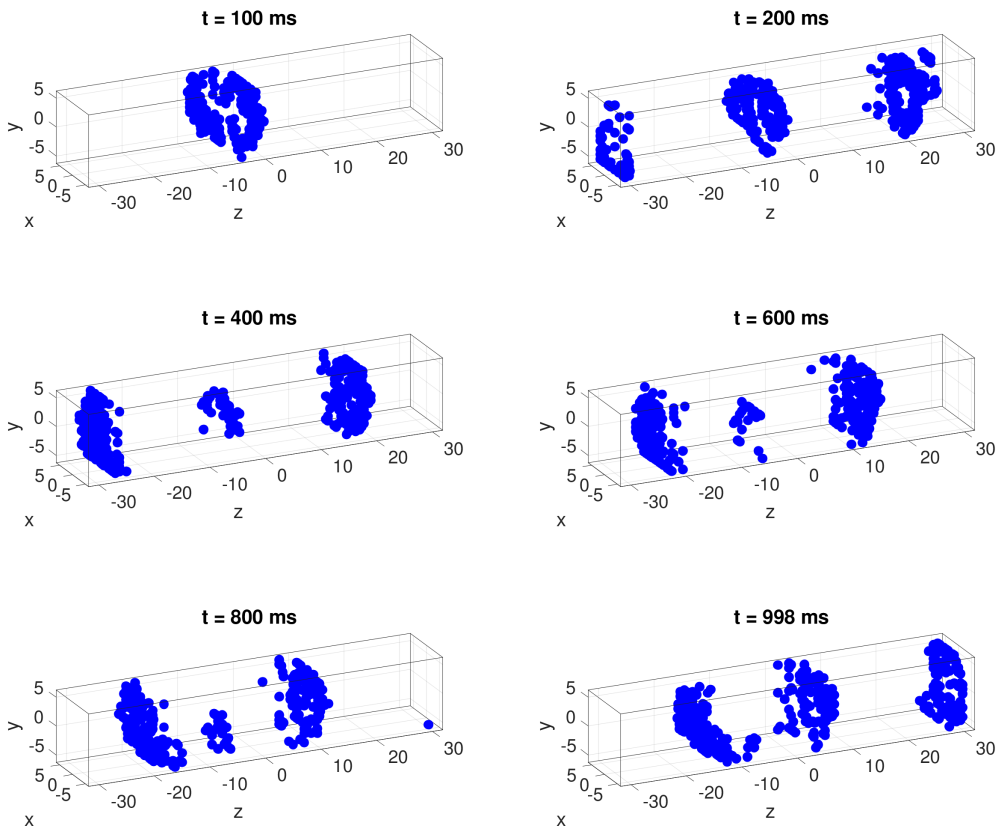

Figure 2. Open calcium release units throughout the cell.
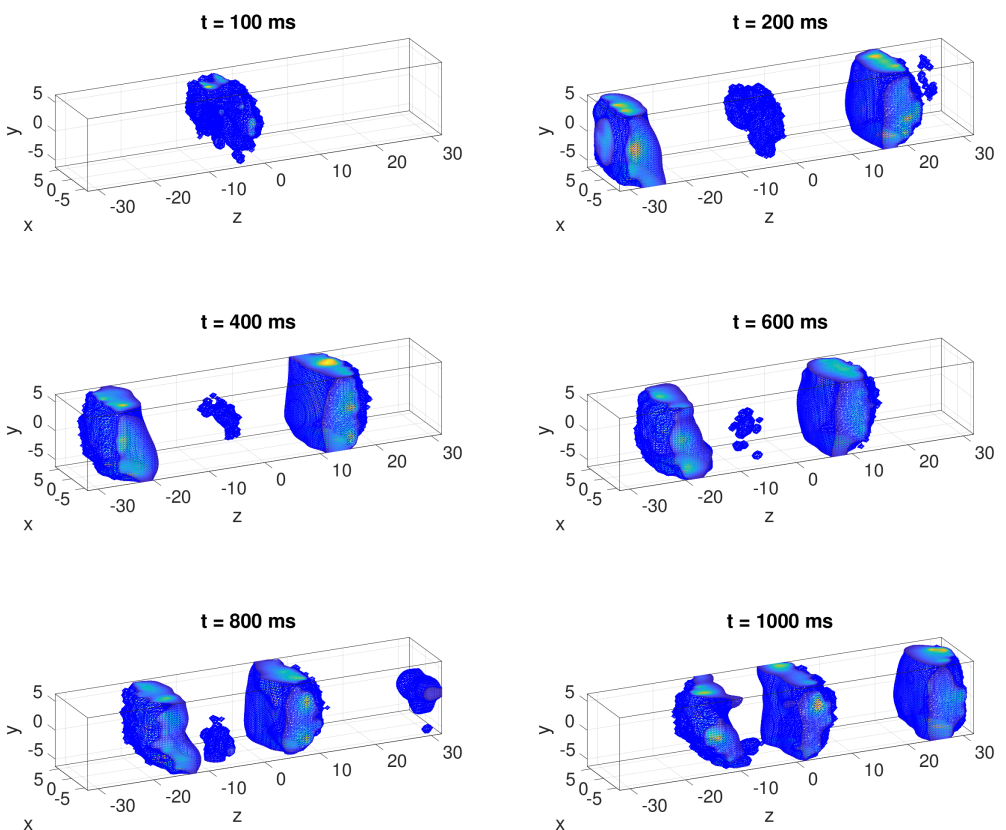

Figure 3. Concentration of $c(\mathbf{x}, t)$ with a critical value of $65 \mu \mathrm{M}$. 
4.2. Adding the Calcium Store in the Sarcoplasmic Reticulum (SR). To add the calcium store in the Sarcoplasmic Reticulum (SR), (5) must provide nonconstant concentrations of calcium. We can set $\gamma=14$ to remove the previous reduction of this equation in Subsection 4.1. These values for $\gamma$ and $D_{s}$ are from Table 5.

We can look at the isosurface plots for calcium in the SR to determine the functionality of the calcium store in the SR with the two changed parameters. The seven equations allows for a range of concentrations for calcium in the SR from 0.01 to $10,000 \mu \mathrm{M}$. Figure 4 shows a collection of isosurface plots for calcium concentration in the $\mathrm{SR}$ with a critical value of $5,000 \mu \mathrm{M}$. Concentrations more extreme than the critical value are represented with a spectrum start from blue to yellow, where yellow represents concentrations closest to the maximum. When looking at Figure 4 the lighter concentrations of calcium in the SR directly correspond to the extreme concentrations of calcium throughout the cell.

Figure 4 shows that the calcium store was implemented. Unlike in Subsection 4.1 where the concentration of calcium in the SR remained constant at $10,000 \mu \mathrm{M}$, the subplots in Figure 4 contain numerous examples of varying concentration of calcium in the SR.

Setting $\gamma=14$ was determined through an analysis of several other cases with different values for $\gamma$. From the range that was tested, 0.01 to 1000 , every value eventually resulted in continuous calcium waves. This is expected as adding the caclium store does not hinder the continuous calcium waves. However, $\gamma$ values 100 and above resulted in a range of calcium concentration in the SR that included negative values. The seven equation model is limited for large $\gamma$ values as it does not account for negative concentrations, which physiologically are inconceivable. Simulations for $\gamma$ values 1 and below resulted in insignificant changes to the calcium in the SR, showing that the smaller values of gamma do not sufficiently add the decrease in calcium from the store according to the increase of calcium in the cytosol. From the parameter study, $\gamma=10$ was idealistic as continuous calcium waves are apparent and an appropiate calcium store was successfully implemented. In this paper, $\gamma=14$ is used to keep consistency with previous studies that used $\gamma=10$. No significant differences can be found between $\gamma=14$ and $\gamma=10$.

The CRU plots and the isosurface plots for $c(\mathbf{x}, t), b_{1}^{(c)}(\mathbf{x}, t)$, and $b_{2}^{(c)}(\mathbf{x}, t)$ remained nearly identical to the plots in Subsection 4.1 This shows that the implementation of the calcium store did not heavily influence the concentrations of free flourescent dye from (2) and free troponin from (3). 

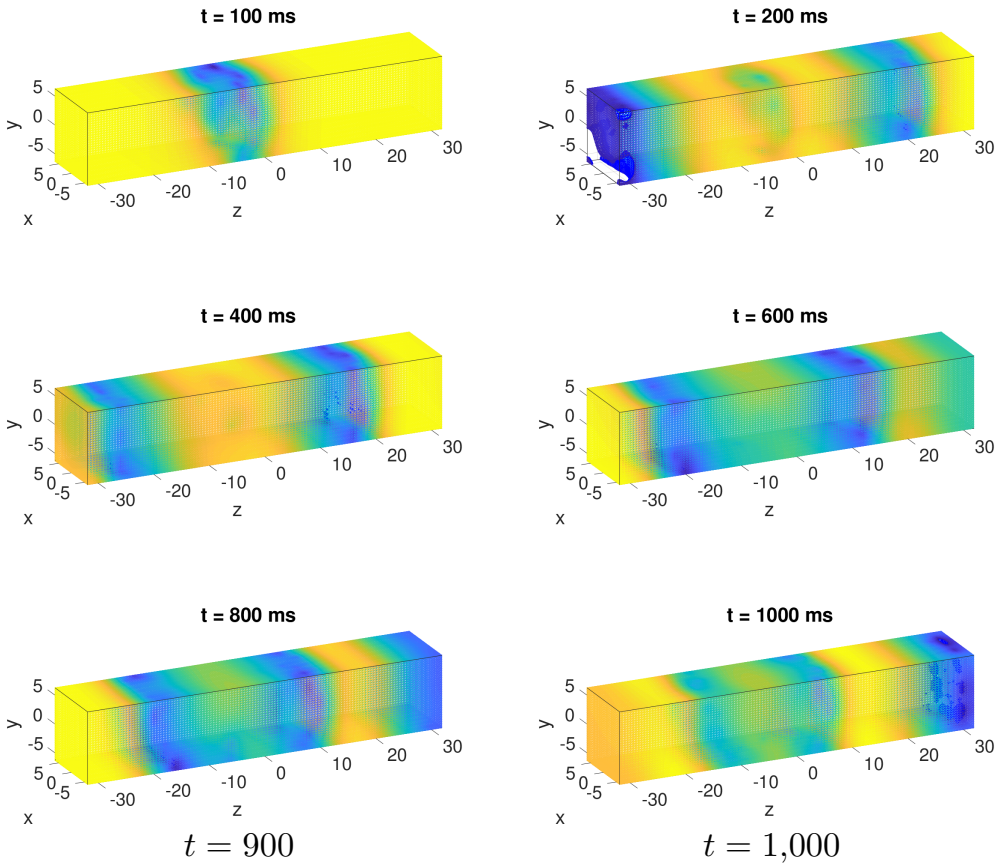

Figure 4. Concentration of $s(\mathbf{x}, t)$ with a critical value of $5000 \mu \mathrm{M}$. 
4.3. Adding the Effect of Electrical Excitation. Implementing the effect of electrical excitation on top of the calcium store in the SR is achieved through activating (6) and (7). From the reduced version of the seven variable model, setting $\tau_{v}=0.1 \mu \mathrm{M} \mu \mathrm{m}^{3} / \mathrm{ms}, V_{m_{\text {pump }}}=0.3 \mu \mathrm{M} / \mathrm{ms}, \omega=100$, and $\kappa=0.1$ results in functional equations for electrical excitation. We can make several other cases with various values of each varied parameter to see how different extremes for each variable changes the resulting calcium wave.

From Subsection 4.2, we performed three separate one-dimensional studies in order to find values for $V_{m_{\text {pump }}}, \omega$, and $\kappa$ that fully add the voltage system without significant changes to the various species concentrations in Subsection 4.2.

The first one-dimensional study varied $V_{m_{p u m p}}$ from 0.001 to 1 . Changing this parameter a non-zero value allows for the functionality of the function (21) and consequently (22). Both $V_{m_{\text {pump }}}$ and $V_{m_{\text {leak }}}$ have an influence on equations (1) and (6). From this study, the largest value that retains the continous calcium waves described in Subsection 4.1 was $V_{m_{\text {pump }}}=0.3 \mu \mathrm{M} / \mathrm{ms}$.

With this fixed value for $V_{m_{\text {pump }}}$, we can perform a one dimensional study of $\omega$, the feedback strength for $\mathrm{Ca}^{2+}$ efflux, and following an additonal study of $\kappa$. When varying $\omega$, we noted that from $\omega=0.1$ to 1000 , the CRU plots are almost identical to our CRU plots from Subsection 4.2. We this as such, can set $\omega=100.0$, a fixed value, as all tested cases of $\omega$ simulated similar results to the plots shown in Subsection 4.2 .

To fully implement the voltage system, we need to test values of $\kappa$ to find what nonzero value of $\kappa$ would allow for continous calcium waves with a fully implemented voltage system and calcium in the SR. When varying $\kappa$ from 0.001 to 10 , we noted that $\kappa=0.1$ was the largest value in which the calcium waves were comprable to the previous subsections.

Setting $\kappa=0.1, \omega=100, \tau_{v}=0.1 \mu \mathrm{M} \mu \mathrm{m}^{3} / \mathrm{ms}$, and $V_{m_{\text {pump }}}=0.3 \mu \mathrm{M} / \mathrm{ms}$ in addition to the predetermined values for the parameters in Table 5 allows for the full implementation of the voltage system without fundamentally changing the outflow of calcium.

The CRU plots and the isosurface plots for $c(\mathbf{x}, t), b_{1}^{(c)}(\mathbf{x}, t)$, and $b_{2}^{(c)}(\mathbf{x}, t) \operatorname{did}$ not significantly change with the implementation of the voltage system. However, there are more notable differences when comparing the calcium concentrations of the stores shwon in Figure 5 and Figure 4. In Figure 5, the latter plots have colorless space between each clump of calcium. As these spaces are representative of lower calcium concentrations, these plots show that the store in the SR has lower concentrations of calcium. Larger time studies may indicate that the calcium concentration in the store continues to lower past $t=100 \mathrm{~ms}$.

The voltage plot in Figure 6 shows that the electrical system was implemented. The voltage plots from the previous subsections remained constant at $-50 \mathrm{mV}$. In Figure 6, there is a clear repetition of the voltage rising up to approximately 26 $\mathrm{mV}$ back down to approximately $-23 \mathrm{mV}$. This figure confirms that external voltage is present. Based on these comparisions, these plots maintain the same behaviors from Subsection 4.2 with the addition of the voltage system. 

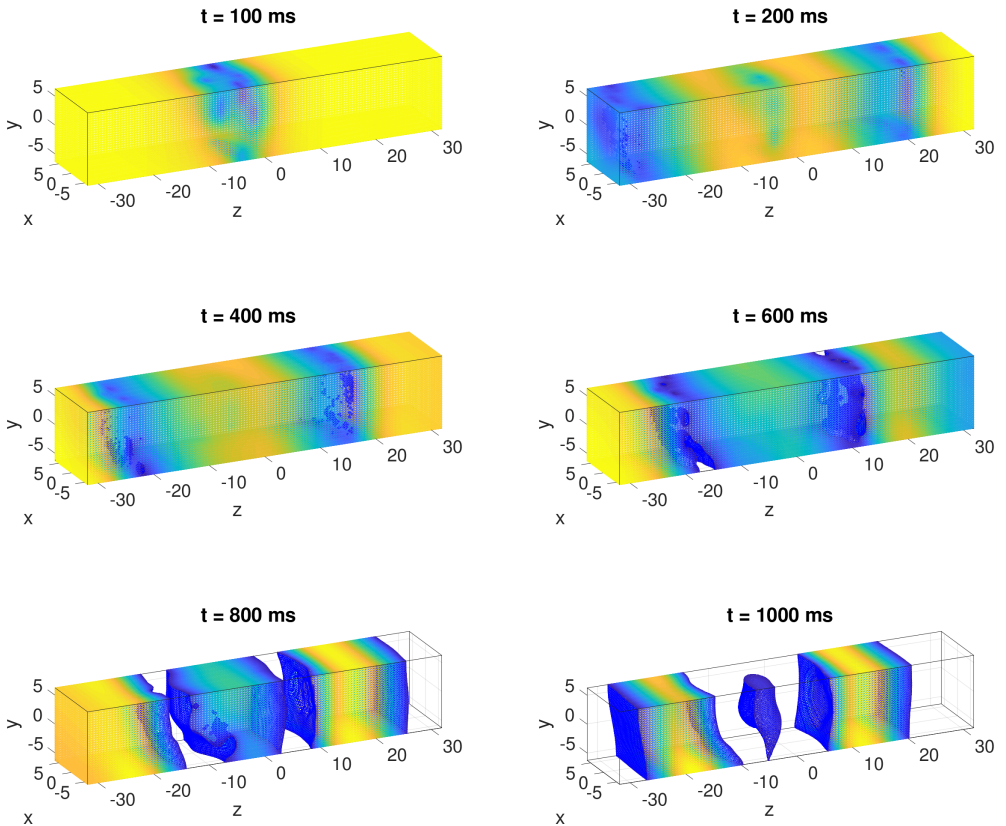

Figure 5. Concentration of $s(\mathbf{x}, t)$ with a critical value of $5000 \mu \mathrm{M}$.

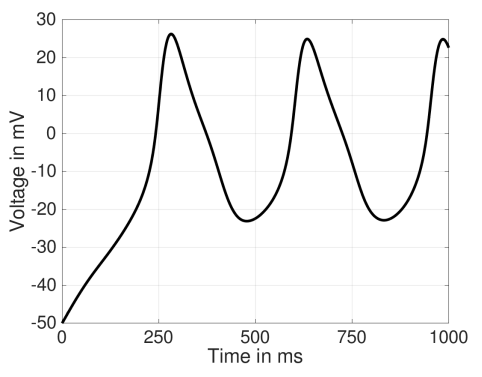

Figure 6. Voltage levels throughout the cell over period of $1000 \mathrm{~ms}$ 
4.4. Adding the Effect of Mechanical Contraction. In the seven variable model, mechanical contraction is described through (3) and (4). From the parameters added in Subsection 4.3 shown in Table 5, a parameter study was performed on the values for $F_{\max }$. By changing this parameter to nonzero values, we can study the effects of mechanical contraction on top of the previously included calcium store and electrical excitation. In additon to these set values, we created other cases with various values for $F_{\max }$ to note the physiological changes with various values of (3) and (4).

For this parameter study, we tested a range from $F_{\max }=0.001$ to $F_{\max }=100$. For $F_{\max }$ values less than 1, there shortening factor remained the same, where after about $125 \mathrm{~ms} \varepsilon$ began to cycle between a small range and remained this way up to $1000 \mathrm{~ms}$. For $F_{\max }=100$, the shortening factor loses its period, where the value does not cycle. Rather, the shortening factor spikes up two times throughout the 1,000 ms. While $F_{\max }=10$ maintains the cyling value of the shortening factor, there is an apparent delay for the cycle to begin. Unlike the start time of $125 \mathrm{~ms}$ for the lower values of $F_{\max }$, for $F_{\max }=10$, the start time is $250 \mathrm{~ms}$. Since the simulations are only 1,000 ms in length the desired value for $F_{\max }$ is 1 .

As in the previous subsections, the CRU plots and the isosurface plots for $c(\mathbf{x}, t)$, $b_{1}^{(c)}(\mathbf{x}, t)$, and $b_{2}^{(c)}(\mathbf{x}, t)$ appear to be extremely similar if not identical to plots from the previous subsections. Additionally, the concentrations shown in Figures 5 and 6 remain the same with the addition of $F_{\max }$ This shows that the implementation of the mechanical contraction did not heavily influence the other components to the extent of changing the system.

With $F_{\max }$ set to a nonzero value, the shortening factor, described in (11), is able to vary based on inactive actin-myosin cross-bridges described in (4). In the previous subsections, the lack of the variance in the shortening factor led to inability for the continuous contractions of the cross-bridges to take place. Unlike the previous subsections, this shortening factor is able to vary in severity allowing for the repetition of inactive cross-bridges switching to active ones. This repetitive cycle is displayed in Figure 7. In previous subsections, the plots for inactive actinmyosin cross-bridges remained constant throughout the 1,000 ms.

As in the previous subsections, the CRU plots and the isosurface plots for $c(\mathbf{x}, t)$, $b_{1}^{(c)}(\mathbf{x}, t)$, and $b_{2}^{(c)}(\mathbf{x}, t)$ appear to be extremely similar to plots from the previous subsections. Additionally, the concentrations shown in Figures 5 and 6 remain the same with the addition of $F_{\max }$ This shows that the implementation of the mechanical contraction with the so-far chosen parameters did not heavily influence the other components to the extent of changing the system. 

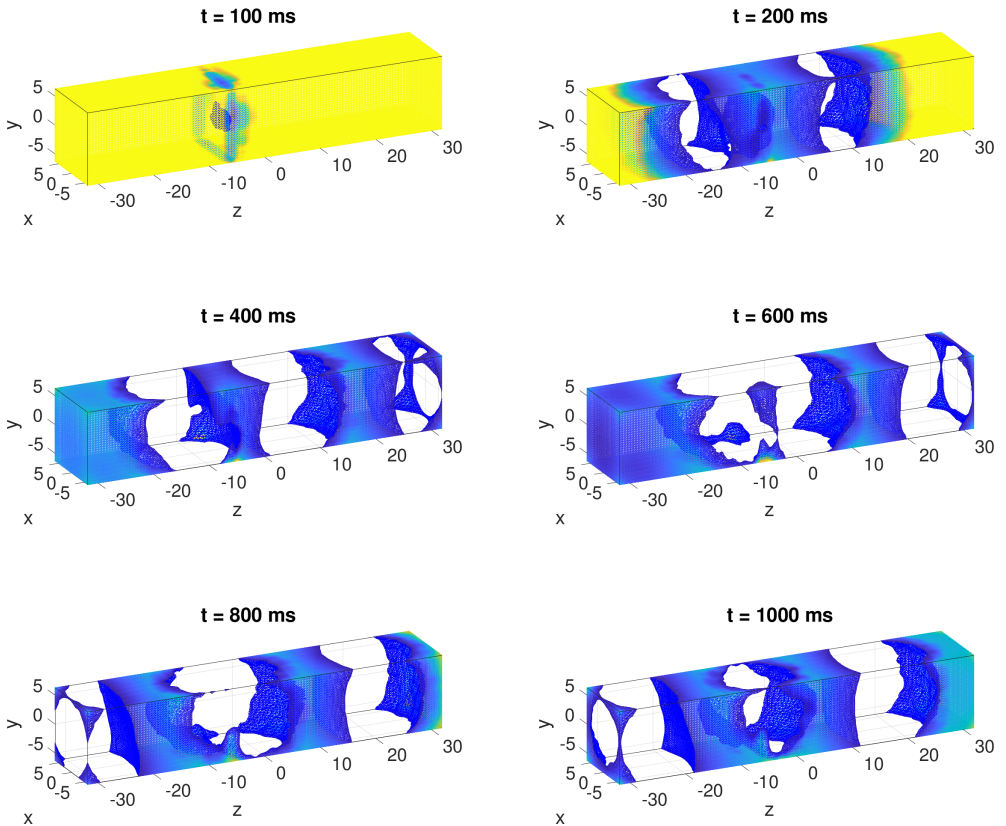

Figure 7. Concentration of $b_{3}^{(c)}(\mathbf{x}, t)$ with a critical value of $50 \mu \mathrm{M}$. 
4.5. Influence of $F_{\max }$ on the Entire System. With these set parameters described in Subsection 4.1, we are able to test questions retaining to the entire model. One example of this is the study of $F_{\max }$ for the much longer final time $t_{\text {fin }}=5,000 \mathrm{~ms}$ rather than $1,000 \mathrm{~ms}$ on the seven variable model. From the tested values for $F_{\max }$ from 0.001 to 100 , values 1 and 100 are shown in the plots below. These values were determined through the parameter study of $F_{\max }$ from Subsection 4.4 .

Figures 8 and 12 show plots of both the voltage $V$ and shortening factor $\varepsilon$ vs. time for the model. While the content is different, the structure of the voltage plots is identical to the voltage plots described in Subsection 4.3. The shortening factor $\varepsilon$ vs. time plots track the value of the shortening factor, $\varepsilon$, used in (3).

There are several differences between how each $F_{\max }$ value affects the system. Figures 8-11 are representative of the same simulations as in Subsection 4.4, except the duration of time was extended from $t_{f} i n=1,000 \mathrm{~ms}$ to $5,000 \mathrm{~ms}$. As previously described, around $125 \mathrm{~ms}$ the calcium concentration in the cytosol shown in Figure 9 jumps from 0 to slightly less than $4 \times 10^{5}$ and slowly decreases as the calcium concentration in the store depletes. In Figure 11, the calcium concentration in the store drops at the same time. The calcium concentration in the store continues to drop until about 2,500 ms, where it then levels out. The concentration of $b_{3}^{(c)}(\mathbf{x}, t)$ in Figure 10 drop at the same time as the calcium change in the cytosol. Once the calcium in the cytosol depletes, the concentration of $b_{3}^{(c)}(\mathbf{x}, t)$ returns to its initial state.

When comparing Figures 8-11 using $F_{\max }=1$ and Figures $12-15$ using $F_{\max }=$ 100, there are clear differences. In Figure 13, the calcium concentration jumps from its initial state similarly to the calcium concentration of Figure 9. However, it shortly returns to its initial state and remains so for several hundred milliseconds before jumping back up. This spike back downards is not characterized in Figures 811. The concentration around $1,000 \mathrm{~ms}$ jumps up to nearly $9 \times 10^{5}$, more than double the peak concentration of calcium in the cytosol for $F_{\max }=1$. Furthermore, the range of concentration levels in $100 \mathrm{~ms}$ is much larger than in Figure 9. The calcium in the store, shown in Figure 11, overall has a downard trend until around 3,000 ms, where the calcium concentration in the store levels out. The calcium concentration in the cytosol in Figure 9 correspondingly drops down to nearly 0. Similar to the first spike of calcium in Figure 9, in Figure 10, the concentration of $b_{3}^{(c)}(\mathbf{x}, t)$ drops with the spike and almost returns to its initial state before dropping a second time around 1,000 ms. The concentration remains relatively constant until the calcium in the cytosol drops. At this point the concentration of $b_{3}^{(c)}(\mathbf{x}, t)$ rises back up to the same concentration as its initial state and remains around that level until the final time $t_{f i n}=5,000 \mathrm{~ms}$.

There are also notable differences between the plots of the shortening factor $\varepsilon$ vs. time in Figures 8 and 12. Both plots generally have the same characteristics as the plots of $b_{3}^{(c)}$. Where the value for $\varepsilon$ is greater, the concentration of $b_{3}^{(c)}$ is lower. Between the plots in Figures 8 and 12, the range of oscillation is much smaller in Figure 12 than in Figure 8 during the period where the value of $\varepsilon$ oscillates.

Unlike the other plots, the plots of voltage $V$ vs. time in Figures 8 and 12 retain consistency throughout the entire 5,000 ms. The voltage level in both plots oscilates from a negative value to a positive value. It appears that the range of one oscillation of voltage shrinks slightly as the calcium levels in the cytosol increase. Other than 
this, it appears that the change in $F_{\max }$ does not have such a drastic impact on the characteristics of voltage compared to the concentrations of each species of the cell. As such, it appears that the influence of the mechanical system is directed towards calcium signaling and therefore has an indirect influence on voltage. This might be expected as there are no direct links between the mechanical system and electrical excitation. 

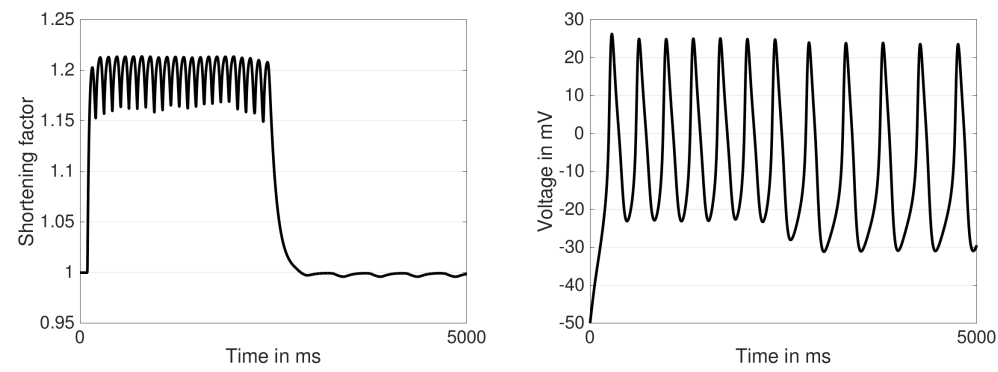

Figure 8. Plots of voltage $V$ and shortening factor $\varepsilon$ vs. time with $F_{\max }=1$ for $t_{f i n}=5,000 \mathrm{~ms}$.

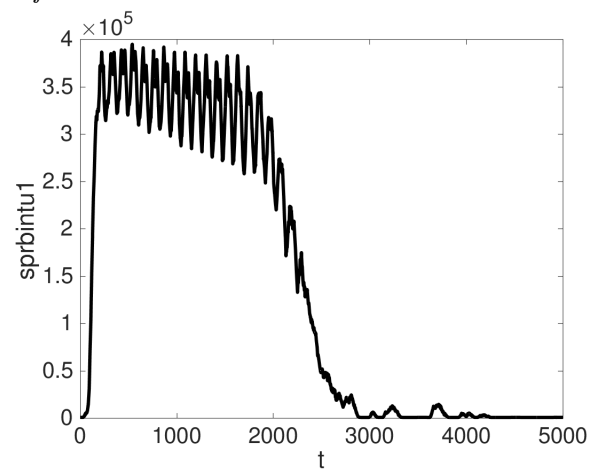

FiguRE 9. Total concentration of $c(\mathbf{x}, t)$ vs. time with $F_{\max }=1$ for $t_{\text {fin }}=5,000 \mathrm{~ms}$.

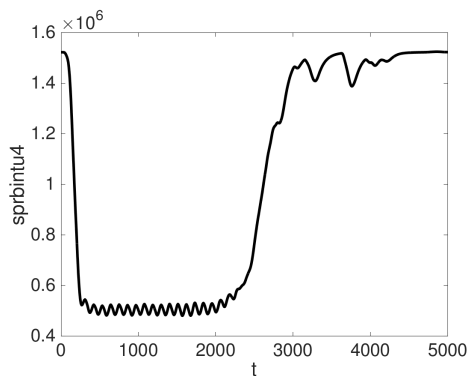

Figure 10. Total concentrations of $b_{3}^{(c)}(\mathbf{x}, t)$ vs. time with $F_{\max }=$ 1 for $t_{\text {fin }}=5,000 \mathrm{~ms}$.

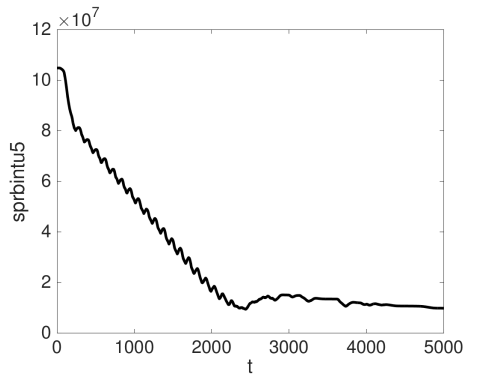

Figure 11. Total concentrations of $s(\mathbf{x}, t)$ vs. time with $F_{\max }=1$ for $t_{\text {fin }}=5,000 \mathrm{~ms}$. 

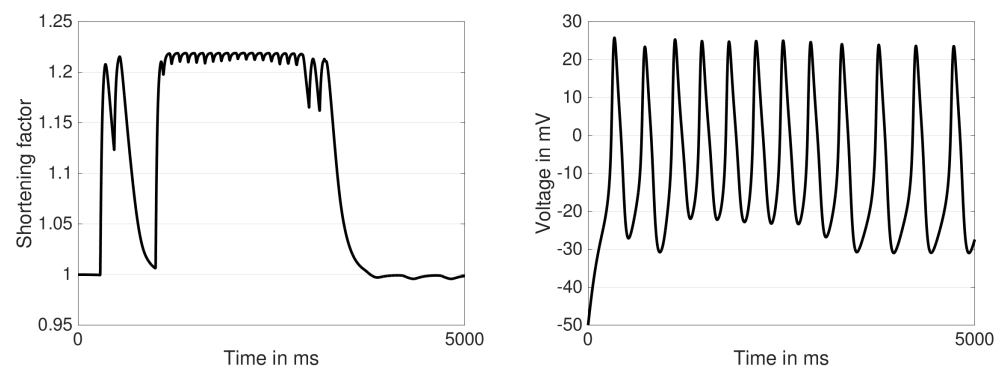

Figure 12. Plots of voltage $V$ and shortening factor $\varepsilon$ vs. time with $F_{\max }=100$ for $t_{\text {fin }}=5,000 \mathrm{~ms}$.

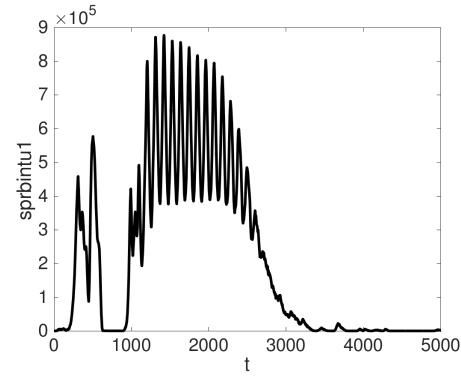

Figure 13. Total concentration of $c(\mathbf{x}, t)$ vs. time with $F_{\max }=$ 100 for $t_{\text {fin }}=5,000 \mathrm{~ms}$.

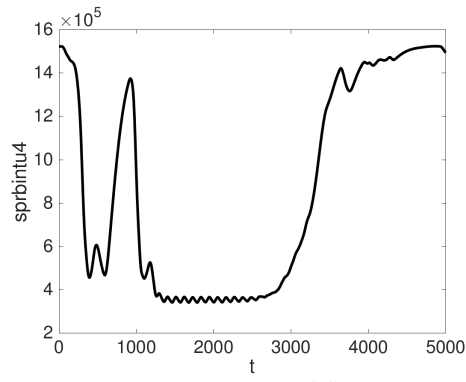

FiguRE 14. Total concentrations of $b_{3}^{(c)}(\mathbf{x}, t)$ vs. time with $F_{\max }=$ 100 for $t_{\text {fin }}=5,000 \mathrm{~ms}$.

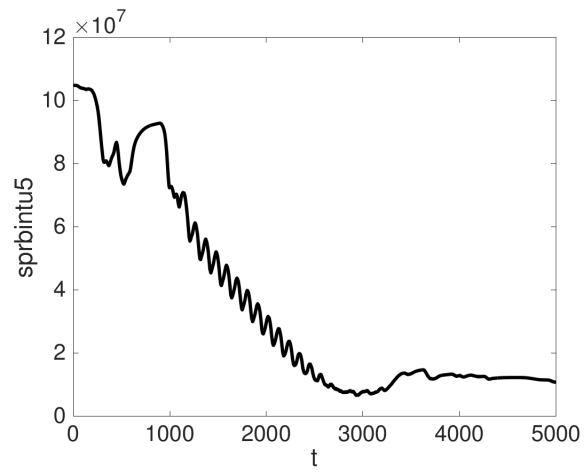

Figure 15. Total concentrations of $s(\mathbf{x}, t)$ vs. time with $F_{\max }=$ 100 for $t_{\text {fin }}=5,000 \mathrm{~ms}$. 


\section{Conclusions And Outlook}

We successfully created a table of parameters in Section 4 that allow for a complete functioning model. This model includes the calcium store, electrical excitation, and mechanical contraction of the cardiac muscle cell. Unlike before, this table allows for the cohesion of all components of the model. With this, we are able to study the model in its entirety. As an example, we showed some influences of $F_{\max }$, a constant of the shortening factor, on the entire system over a period of 5,000 ms. When comparing a small and large value for $F_{\max }$, we noticed that the influence of $F_{\max }$ was much less extreme on the electrical excitation compared to the calcium signaling. When comparing the plots of the relevant species of the system, this conclusion becomes apparent. This is important as it allows us to grasp a better understanding of the linkage between the mechanical system and calcium signaling, shown by Link (1) in Figure 1.

Future research on this could look more in depth at different values of other parameters with a final time of $5,000 \mathrm{~ms}$. This would allow for studies on the system for longer periods of time. Studying multiple cases for each parameter value with randomized locations for calcium sparking would allow for more concrete conclusions with a stronger representation of the physiological processes of the cell. While this is one further area of study, this complete set of parameters opens many areas to study the model in its entirety.

\section{ACKNOWLEDGEMENTS}

The hardware in the UMBC High Performance Computing Facility (HPCF) is supported by the U.S. National Science Foundation through the MRI program (grant nos. CNS-0821258, CNS-1228778, and OAC-1726023) and the SCREMS program (grant no. DMS-0821311), with additional substantial support from the University of Maryland, Baltimore County (UMBC). See hpcf . umbc . edu for more information on $\mathrm{HPCF}$ and the projects using its resources. Co-author Carlos Barajas was supported by UMBC as HPCF RA. 


\section{REFERENCES}

$\left[\mathrm{ABM}^{+} 16\right]$ Kallista Angeloff, Carlos A. Barajas, Alexander Middleton, Uchenna Osia, Jonathan S. Graf, Matthias K. Gobbert, and Zana Coulibaly. Examining the effect of introducing a link from electrical excitation to calcium dynamics in a cardiomyocyte. Spora: A Journal of Biomathematics, 2:49-73, 2016.

$\left[\mathrm{ADFI}^{+} 15\right]$ Amanda M. Alexander, Erin K. DeNardo, Eric Frazier III, Michael McCauley, Nicholas Rojina, Zana Coulibaly, Bradford E. Peercy, and Leighton T. Izu. Spontaneous calcium release in cardiac myocytes: Store overload and electrical dynamics. Spora: A Journal of Biomathematics, 1:36-48, 2015.

$\left[\mathrm{BHJ}^{+}{ }^{12}\right]$ Tamas Banyasz, Balazs Horvath, Zhong Jian, Leighton $\mathrm{T}$ Izu, and Ye Chen-Izu. Profile of L-type $\mathrm{Ca} 2+$ current and $\mathrm{Na}+/ \mathrm{Ca} 2+$ exchange current during cardiac action potential in ventricular myocytes. Heart Rhythm, 9(1):134-142, 2012.

$\left[\mathrm{DFL}^{+} 17 \mathrm{a}\right]$ Kristen Deetz, Nygel Foster, Darius Leftwich, Chad Meyer, Shalin Patel, Carlos Barajas, Matthias K. Gobbert, and Zana Coulibaly. Developing the coupling of the mechanical to the electrical and calcium systems in a heart cell. Technical Report HPCF-2017-15, UMBC High Performance Computing Facility, University of Maryland, Baltimore County, 2017.

$\left[\mathrm{DFL}^{+} 17 \mathrm{~b}\right]$ Kristen Deetz, Nygel Foster, Darius Leftwich, Chad Meyer, Shalin Patel, Carlos Barajas, Matthias K. Gobbert, and Zana Coulibaly. Examining the electrical excitation, calcium signaling, and mechanical contraction cycle in a heart cell. Spora: A Journal of Biomathematics, 3:66-85, 2017.

[Gob08] Matthias K. Gobbert. Long-time simulations on high resolution meshes to model calcium waves in a heart cell. SIAM J. Sci. Comput., 30(6):2922-2947, 2008.

[HGI04] Alexander L. Hanhart, Matthias K. Gobbert, and Leighton T. Izu. A memory-efficient finite element method for systems of reaction-diffusion equations with non-smooth forcing. J. Comput. Appl. Math., 169(2):431-458, 2004.

[IMBW01] Leighton T. Izu, Joseph R. H. Mauban, C. William Balke, and W. Gil Wier. Large currents generate cardiac $\mathrm{Ca}^{2+}$ sparks. Biophys. J., 80:88-102, 2001.

[IWB01] Leighton T. Izu, W. Gil Wier, and C. William Balke. Evolution of cardiac calcium waves from stochastic calcium sparks. Biophys. J., 80:103-120, 2001.

$\left[\mathrm{M}^{+} 14\right]$ Benjamin Mozaffarian et al. Heart disease and stroke statistics - 2015 update: A report from the american heart association. Circulation, 131(4):e29, 2014.

[ML81] Catherine Morris and Harold Lecar. Voltage oscillations in the barnacle giant muscle fiber. Biophys. J., 35(1):193, 1981.

[SGTK12] Thomas I. Seidman, Matthias K. Gobbert, David W. Trott, and Martin Kružík. Finite element approximation for time-dependent diffusion with measure-valued source. Numer. Math., 122(4):709-723, 2012.

$\left[\mathrm{SHK}^{+} 15\right]$ Jonas Schäfer, Xuan Huang, Stefan Kopecz, Philipp Birken, Matthias K. Gobbert, and Andreas Meister. A memory-efficient finite volume method for advectiondiffusion-reaction systems with non-smooth sources. Numer. Methods Partial Differential Equations, 31(1):143-167, 2015. 